1995

\title{
Stability and Resolution in Thermal Imaging
}

Lester Caudill

University of Richmond, lcaudill@richmond.edu

Kurt Bryan

Follow this and additional works at: http://scholarship.richmond.edu/mathcs-faculty-publications

Part of the Health Information Technology Commons, and the Mathematics Commons

\section{Recommended Citation}

Caudill, Lester, and Kurt Bryan. "Stability and Resolution in Thermal Imaging." Proceedings of the ASME Design Engineering Technical Conferences 3 (1995): 1023-1032.

This Article is brought to you for free and open access by the Math and Computer Science at UR Scholarship Repository. It has been accepted for inclusion in Math and Computer Science Faculty Publications by an authorized administrator of UR Scholarship Repository. For more information, please contact scholarshiprepository@richmond.edu. 


\title{
STABILITY AND RESOLUTION IN THERMAL IMAGING ${ }^{1}$
}

\author{
Kurt Bryan \\ Department of Mathematics \\ Rose-Hulman Institute of Technology \\ Lester F. Caudill, Jr. \\ Department of Mathematics \\ University of Kentucky
}

\begin{abstract}
This paper examines an inverse problem which arises in thermal imaging. We investigate the problem of detecting and imaging corrosion in a material sample by applying a heat flux and measuring the induced temperature on the sample's exterior boundary. The goal is to identify the profile of some inaccessible portion of the boundary. We study the case in which one has data at every point on the boundary of the region, as well as the case in which only finitely many measurements are available. An inversion procedure is developed and used to study the stability of the inverse problem for various experimental configurations.
\end{abstract}

\section{Introduction}

Some of the fastest growing areas of non-destructive evaluation (NDE) are those related to the assessment of the condition of aging aircraft. Thermal imaging is a technique that has shown promise for detecting corrosion or delaminations in aircraft. The technique is used to recover information about the internal condition of a sample by applying a heat flux to its boundary and observing the resulting temperature response on the object's surface. From this information, one attempts to determine the internal thermal properties of the object, or the shape of some unknown (possibly corroded) portion of the boundary. Patel et al. (1992) provide account of the technology and typical data processing

\footnotetext{
${ }^{1}$ This research was partially carried out while the first author was in residence at the Institute for Computer Applications in Science and Engineering (ICASE), NASA Langley Research Center, Hampton, VA 23681, which is operated under National Aeronautics and Space Administration contract NAS1-19480.
}

techniques that are employed, and a more extensive bibliography on the subject.

One of the most common uses for thermal imaging is for the detection of so-called "back surface" corrosion and damage. Briefly, one attempts to determine whether some inaccessible portion of an object's boundary has corroded, and therefore changed shape. In this paper we investigate a model two-dimensional version of the problem, to gain some insight into the nature of the mathematics involved, especially the structure and conditioning of the mathematical inverse problem. We consider a certain portion of the surface of a rectangular sample to be accessible for measurements and the remainder of the surface, which may be corroded, inaccessible. This problem has been considered by others (Banks et al., 1989, 1990) with an emphasis on recovering estimates of the unknown surface from data by using an output least-squares method.

We examine both a continuous and finite data version of the inverse problem. The continuous version assumes that one has data at every point on the accessible portion of the object's surface. The finite data version assumes that only finitely many measurements have been made. Our goals are

- To determine whether it is in principle possible to recover the back surface from data, and examine the sensitivity of the inverse problem to noise in the data.

- To examine how various experimental parameters affect stability and resolution for the inverse problem, especially the effect of measurement locations on stability.

- To determine how one might incorporate a priori information or assumptions into the inverse problem.

Our main focus is not to develop inversion algorithms, but in the course of examining the problem, we derive 
an inversion procedure for the finite data inverse problem. This algorithm allows the easy incorporation of a priori assumptions into the inversion process. We apply the algorithm to several simulated data sets to illustrate our conclusions. Our study of the stability of the inverse problem reduces to studying the invertibility of a certain matrix, which we do with a singular value decomposition. We do not make any explicit finite dimensional parameterization of the unknown surface.

We should note that a very similar approach has been used by Dobson and Santosa (1994) to study resolution and stability for the inverse conductivity problem. Isaacson et al. (1990a, 1990b) have also carried out similar sensitivity studies related to the inverse conductivity problem, especially the effect of finitely many measurements on the inversion process.

The outline of the paper is as follows. In Section 2 we present the mathematical formulation of the continuous and finite data versions of the inverse problem. In Section 3 we derive a linearized version of the inverse problem and show how this leads (as thermal inverse problems often do) to a first kind integral equation which must be inverted. We also state some uniqueness and stability results for the linearized version of the inverse problem. In Section 4 we consider an algorithm for solving the finite data version of the inverse problem and how this approach can be used quantify the stability of the problem. Finally, we present numerical studies to examine the effects that various experimental parameters have on the stability and resolution of the inversion process, and the effect of incorporating a priori assumptions into the inversion procedure.

\section{The Inverse Problem}

Consider a sample to be imaged as a two-dimensional region $\Omega$ lying between the two surfaces $x_{2}=S\left(x_{1}\right)$ and $x_{2}=1$ as illustrated below.

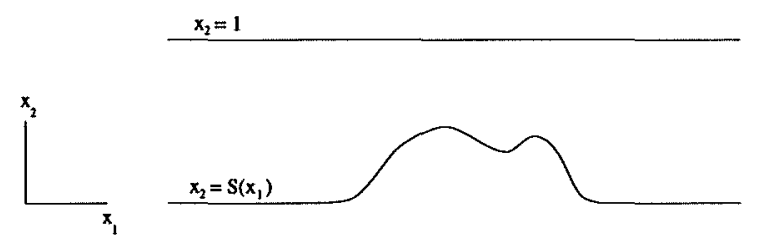

Figure 1: Sample geometry.

The surface $x_{2}=1$ is the "top" or "front" surface and $x_{2}=S\left(x_{1}\right)$ is the "back" surface. We assume that the ends of the sample are sufficiently far away that they can be ignored, so for our purposes the sample is unbounded in the $x_{1}$ direction. The top surface is accessible for inspection and measurements, but the back surface $x_{2}=S\left(x_{1}\right)$ is inaccessible. This is the portion of the sample to be inspected for corrosion. The ideal uncorroded case is a flat back surface $S\left(x_{1}\right) \equiv 0$. In the corroded case illustrated in Figure 1, $S\left(x_{1}\right)>0$ for some values of $x_{1}$. We will assume that the function $S$ belongs to $H^{2}(\mathbb{R})$, although this assumption will later be relaxed. In particular, since $H^{2}(\mathbb{R}) \subset C^{1}(\mathbb{R})$ there is a continuous unit normal vector field on the back surface. The goal is to determine the back surface or the function $S$ by taking measurements only on the front surface.

A time-dependent heat flux $g\left(x_{1}, t\right)$ is applied to the top of the sample $x_{2}=1$. We assume that the sample material is homogeneous with thermal diffusivity $\kappa$ and thermal conductivity $\alpha$, both known constants. We will use $T(x, t)$ to denote the resulting temperature induced in $\Omega$, where $x=\left(x_{1}, x_{2}\right)$. The direct thermal diffusion problem will be modeled as

$$
\begin{aligned}
\frac{\partial T}{\partial t}-\kappa \Delta T & =0 \text { in } \Omega \\
\alpha \frac{\partial T}{\partial \nu} & =g\left(x_{1}, t\right) \text { on } x_{2}=1 \\
\alpha \frac{\partial T}{\partial \nu} & =0 \text { on } x_{2}=S\left(x_{1}\right) \\
T(x, 0) & =T_{0}(x),
\end{aligned}
$$

for $t>0$, where $\frac{\partial}{\partial \nu}$ denotes the outward normal derivative on the boundary of $\Omega$. The function $T_{0}(x)$ is the initial temperature of the region $\Omega$ at time $t=0$. Note that the back surface is assumed to block all heat conduction.

We consider the useful special case in which the heat flux $g\left(x_{1}, t\right)$ is periodic, of the form $\operatorname{Re}\left[g\left(x_{1}\right) e^{i \omega t}\right]$ with $\omega>0$. Since we are interested in the mathematical structure of the inverse problem, we will for simplicity take the constants $\kappa$ and $\alpha$ equal to one. Under these assumptions the solution to equation (2.1) is given as $T(x, t)=\operatorname{Re}\left[e^{i \omega t} u(x)\right]$ where $u(x)$ satisfies

$$
\begin{aligned}
\Delta u-i \omega u & =0 \text { in } \Omega \\
\frac{\partial u}{\partial \nu} & =g\left(x_{1}\right) \text { on } x_{2}=1 \\
\frac{\partial u}{\partial \nu} & =0 \text { on } x_{2}=S\left(x_{1}\right)
\end{aligned}
$$

at least after transients from the initial temperature have sufficiently decayed. The main case of interest is that in which $g\left(x_{1}\right)$ is constant, corresponding to uniform heating of the outer surface. This is typically the case when heat or flash lamps are used to provide the input flux $g$. For the moment, however, we will not restrict $g$. 
We can consider two versions of the inverse problem, the purely mathematical one in which one measures the temperature at all points on the top surface, and the case in which one has a finite number of measurements. The data need not be actual point measurements of the temperature $u$, but this is the most common situation. Of particular interest are the questions

1. Can the function $S\left(x_{1}\right)$ be uniquely determined by knowing temperature $u\left(x_{1}\right)$ for all $x_{1}$ on the top surface?

2. If $S\left(x_{1}\right)$ is uniquely determined by $u\left(x_{1}\right)$, how sensitive is $S\left(x_{1}\right)$ to perturbations in the data? What kinds of features in the back surface can or cannot be easily determined from the data?

3. Since any practical application falls under the finite data formulation, how stable is the estimate of $S\left(x_{1}\right)$ based on finitely many pieces of data? What factors influence stability in this case, and is there an inversion procedure to produce a reasonable estimate of $S\left(x_{1}\right)$ using finitely many measurements?

The first question is easily answered "yes" by a standard argument. A proof has been given by the authors (1994). Briefly, the uniqueness result is

Suppose $u\left(x_{1}, x_{2} ; S\right)$ denotes the solution to (2.2) with back surface $S$ and nonzero flux $g$. If $u\left(x_{1}, 1 ; S_{1}\right)=$ $u\left(x_{1}, 1 ; S_{2}\right)$ for each $\left(x_{1}, 1\right)$ in an open subset $C$ of the top surface of $\Omega$, then $S_{1}=S_{2}$.

The second and third questions will be examined in the next section by considering a linearization of the original inverse problem.

\section{A Linearization}

We now linearize the original direct problem given by equation (2.2) with respect to the function $S$, and study the inverse problem that arises by using the linearized direct problem. Let $u_{0}(x)$ denote the solution to (2.2) with $S \equiv 0$. The surface $x_{2} \equiv 0$ is a sensible point about which to linearize, since this represents the uncorroded or ideal profile from which we hope to detect any deviation. Let $u_{\epsilon}\left(x_{1}, x_{2}\right)$ denote the solution to (2.2) with back surface $x_{2}=S_{\epsilon}\left(x_{1}\right) \equiv \epsilon S\left(x_{1}\right)$ where $S$ is some fixed $H^{2}(\mathbb{R})$ function and $\epsilon$ is some small number. We use $d_{\epsilon}\left(x_{1}\right)=u_{\epsilon}\left(x_{1}, 1\right)$ for the temperature "data" corresponding to $S_{\epsilon}$ (hence $d_{0}\left(x_{1}\right)=u_{0}\left(x_{1}, 1\right)$ corresponds to $S \equiv 0$ ). We have shown (Bryan and Caudill, 1994) that for the special case of $g \equiv 1$ (uniform heating of the top surface)

$$
d_{\epsilon}\left(x_{1}\right)=d_{0}\left(x_{1}\right)+\epsilon d\left(x_{1}\right)+O\left(\epsilon^{2}\right)
$$

where the function $d\left(x_{1}\right)$ satisfies

$$
d\left(x_{1}\right)=\int_{-\infty}^{\infty} S\left(y_{1}\right) \phi\left(x_{1}-y_{1}\right) d y_{1}=S * \phi
$$

and where " $*$ " denotes convolution. The function $\phi(x)$ is determined uniquely by its Fourier transform $\hat{\phi}(y)$, which is

$$
\hat{\phi}(y)=\frac{C}{\alpha\left(e^{\alpha}-e^{-\alpha}\right)}
$$

where $\alpha=\sqrt{y^{2}-i \omega}$ and the constant $C$ is

$$
C=-\frac{4 i \omega}{\gamma\left(e^{\gamma}-e^{-\gamma}\right)}
$$

with $\gamma=(1-i) \sqrt{\omega / 2}$. The function $\phi(x)$ is analytic and rapidly decreasing (faster than any polynomial); its Fourier transform shares the same properties. Moreover, the function satisfies $\hat{\phi}(y) \neq 0$ for any real value of $y$.

Equation (3.3) is the linearized version of the direct problem; it says that the perturbation in the back surface (about $S \equiv 0$ ) generates a first order perturbation $\epsilon d\left(x_{1}\right)$ in the front surface temperature data, with $d\left(x_{1}\right)$ given by (3.3).

The inverse problem for the linearized direct problem is to identify $S(x)$ given data for the linearized direct problem. Note that the measured data $\epsilon d\left(x_{1}\right)+d_{0}\left(x_{1}\right)$ is equivalent to knowing $d_{\epsilon}$, since $d_{0}$ is in principle known. With $d\left(x_{1}\right)$ considered known (3.3) becomes a first kind integral equation for the unknown function $S$. First kind integral equations have been extensively studied (Tricomi, 1957), (Wing, 1991) and are well-known to be unstable; small perturbations in the right hand side $d(x)$ can lead to arbitrarily large changes in the solution $S$. However this formulation of the inverse problem as an integral equation will allow us to obtain stability estimates for the linearized version of the problem and yields a reasonable approach to reconstruction.

Equation (3.3) shows immediately that the linearized inverse problem has a unique solution. Suppose some surface $S\left(x_{1}\right)$ with $S \in L^{2}(\mathbb{R})$ gives rise to data $d\left(x_{1}\right)$. Fourier transforming both sides of (3.3) and dividing by $\hat{\phi}$ (valid because $\hat{\phi}(y) \neq 0$ ) yields

$$
\hat{S}=\frac{\hat{d}}{\hat{\phi}^{2}}
$$

so $\hat{S}$ can be found in terms of $\hat{d}$. If $S$ is $L^{2}$ then so is $\hat{S}$, and $S$ is determined uniquely by knowledge of $\hat{S}$. Note 
we assumed a priori that $S$ is in $L^{2}(\mathbb{R})$. In general, for an arbitrary $d \in L^{2}(\mathbb{R})$ we cannot find a function $S$ in $L^{2}$ which gives rise to data $d$ via equation (3.3).

The convolution equation (3.3) also provides information on continuous dependence. The function $\hat{\phi}$ is smooth and never equal to zero, and so motivated by equation (3.5), we can define the space of functions $L_{*}^{2}(\mathbb{R})$ with the norm

$$
\|f\|_{*}^{2}=\int_{-\infty}^{\infty}\left|\frac{\hat{f}(z)}{\hat{\phi}(z)}\right|^{2} d z .
$$

From equation (3.4) it follows that $\frac{1}{\hat{\phi}(z)}$ grows like $z e^{z}$. The norm \|\|$_{*}$ thus puts a heavy penalty on high frequencies; the functions in this space are very smooth. Equation (3.5) then shows that

If a back surface $x_{2}=S\left(x_{1}\right)$ generates front surface data $d(x)$ for the linearized direct problem then

$$
\|S\|_{L^{2}} \leq C\|d\|_{*}
$$

where $C$ is independent of $d$.

Estimates of $S$ from data $d$ will thus be extremely sensitive to any noise, because the inversion process weights a frequency $f$ in the data by a factor proportional to $f e^{f}$. The structure of the convolution operator mapping $S$ to the data $d$ makes it clear that it will be difficult to estimate the high spatial frequency components in the Fourier decomposition of $S$, for these components are heavily damped out by the forward mapping.

\section{The Case of Finitely Many Measurements}

Suppose that we have point estimates $d\left(a_{i}\right)=u\left(a_{i}, 1\right)$ of the temperature on the top surface at $n$ distinct points. How can we construct a reasonable estimate of the function $S\left(x_{1}\right)$ ? How can we quantify the stability of the reconstruction with respect to errors in the data, and how does the choice of measurement locations $a_{i}$ affect the stability? Let us assume that we seek an estimate $S \in L^{2}(\mathbb{R})$. Physical considerations make it desirable to obtain an estimate with more regularity, but this will be a consequence of the proposed reconstruction procedure. Based on the convolution equation (3.3) we know that $S$ must satisfy the $n$ constraints

$$
<S, c_{i}>=\int_{-\infty}^{\infty} S\left(x_{1}\right) \bar{c}_{i}\left(x_{1}\right) d x_{1}=d\left(a_{i}\right),
$$

for $i=1, \ldots, n$ with $c_{i}\left(x_{1}\right) \equiv \bar{\phi}\left(a_{i}-x_{1}\right)$ and $<f, g\rangle=\int_{\mathbb{R}} f \bar{g}$ is the usual $L^{2}$ inner product. Note that since $c_{i}$ is an $L^{2}$ function, $S \mapsto<S, c_{i}>$ is a bounded linear functional on $L^{2}$. The set (4.6) is a horribly underdetermined set of equations. We can expect to find an entire translated subspace of functions of codimension $n$ in $L^{2}(\mathbb{R})$ which satisfy the given conditions, and any such function "solves" the inverse problem, in the sense that it gives rise to the measured data.

One practical method for specifying a unique function in $L^{2}$ which solves the inverse problem is to seek that element in $L^{2}$ which satisfies the given conditions and has minimal norm. That such an element exists follows from the fact that the relations (4.6) define a closed convex subset of $L^{2}$ and hence this subset has a unique element of minimal norm. This idea has been used before by Dobson and Santosa (1994) to construct a "pseudoinverse" for the finite measurement case and to characterize the stability and information content for the inverse conductivity problem, and has also been used for reconstruction from partial information in tomographic problems by Byrne and FitzGerald (1982).

It is an easy application of Lagrange multipliers to verify that the unique element of $L^{2}$ with minimum norm which satisfies the constraints (4.6) must be of the form

$$
S\left(x_{1}\right)=\sum_{k=1}^{n} \lambda_{k} c_{k}\left(x_{1}\right)
$$

for some $\left\{\lambda_{k}\right\}_{k=1}^{n} \subseteq \mathbb{C}$. The constants $\lambda_{k}$ can be determined by substituting (4.7) into equations (4.6) and solving the resulting $n \times n$ system. The system is of the form $M \lambda=d$ where $M=\left[m_{i j}\right]$ is an $n$ by $n$ matrix, $\lambda$ is the $n$ vector $\left(\lambda_{1}, \ldots, \lambda_{n}\right)^{T}$ and $d$ is an $n$ vector $\left(d\left(a_{1}\right), \ldots, d\left(a_{n}\right)\right)^{T}$. The entries of $M$ are given by

$$
m_{i j}=\int_{-\infty}^{\infty} \bar{c}\left(x_{1}-a_{i}\right) c\left(x_{1}-a_{j}\right) d x_{1} .
$$

The matrix $M$ is clearly Hermitian and in fact is always invertible if the measurement locations are distinct (Bryan and Caudill, (1994)). Thus this inversion procedure thus always produces a unique estimate of $S$ if the measurement locations are distinct.

We can also "solve" the inverse problem by choosing the unique function $S$ which satisfies equations (4.6) and has minimal norm in a weighted $L^{2}$ space $L_{\delta}^{2}(\mathbb{R})$ with norm defined by the inner product

$$
<f, g>_{\delta}=\int_{-\infty}^{\infty} f\left(x_{1}\right) \bar{g}\left(x_{1}\right) \frac{1}{\delta\left(x_{1}\right)} d x_{1}
$$

where $\delta\left(x_{1}\right)$ is some real-valued non-negative function on $\mathbb{R}$. In this case, we have

$$
\int_{-\infty}^{\infty} S\left(x_{1}\right) c_{i}\left(x_{1}\right) d x_{1}=\int_{-\infty}^{\infty} S\left(x_{1}\right) c_{i}\left(x_{1}\right) \delta\left(x_{1}\right) \frac{d x_{1}}{\delta\left(x_{1}\right)}
$$


where we must assume that $S=0$ wherever $\delta=0$. Thus the integral is understood to be taken only over that set where $\delta$ is non-zero. Equations (4.6) now take the form

$$
<S, c_{i} \delta>_{\delta}=d_{i}
$$

and the minimal norm solution is of the form

$$
S\left(x_{1}\right)=\delta\left(x_{1}\right) \sum_{i=1}^{n} \lambda_{i} c_{i}\left(x_{1}\right) .
$$

The idea is to choose $\delta\left(x_{1}\right)$ to have the same general form as $S\left(x_{1}\right)$, and so incorporate a priori information into the reconstruction based on (4.10) by forcing it to have the same general form. For example, if we know that $S$ is supported in the interval $[-b, b]$ we can choose $\delta(x) \equiv 1$ on $[-b, b]$ and $\delta(x) \equiv 0$ elsewhere. The optimal estimate of $S$ becomes

$$
S(x)=\chi_{[-b, b]} \sum_{i=1}^{n} \lambda_{i} c_{i}(x)
$$

where $\chi_{[-b, b]}$ is the characteristic function of the interval $[-b, b]$ and where the $\lambda_{i}$ are found by solving

$$
\sum_{i=1}^{n}\left(\int_{-b}^{b} c_{i}(x) \bar{c}_{j}(x) d x\right) \lambda_{i}=d_{j}
$$

for $j=1$ to $n$.

\section{Numerical Experiments}

We will now examine the finite data version of the inverse problem by using the previously described inversion procedure. In this section we apply the procedure to simulated data sets, both with and without noise. Our main focus is to examine the stability and resolution of back surface estimates with respect to various experimental parameters, specifically the distribution of the measurement locations along the top surface of the sample. We also demonstrate how a priori assumptions about the nature of the corrosion can be incorporated into the inversion, and the effects such assumptions have on stability and resolution.

In the examples that follow we generate simulated test data using the full direct problem (2.2) with heating $g(x) \equiv 1$. The direct problem is solved by converting it into a boundary integral equation which is then solved numerically. The boundary integral formulation leads to a second kind Fredholm equation; the solution procedure is detailed by the authors elsewhere (Bryan and Caudill, 1994).
To illustrate the general procedure and to show that the inversion algorithm provides reasonable estimates, we begin with a simple example. We apply the inversion procedure to data generated using the back surface

$$
S(x)=\frac{e^{-(x+3)^{2}}}{10}+\frac{e^{-(x+2)^{2}}}{5}+\frac{e^{-4 x^{2}}}{10} .
$$

We use a heating frequency of $\omega=1$. As a first step the functions $c_{i}(x)$ are computed and the matrix $M$ is generated. Since these do not depend on $S$, but only on the geometry and heating frequency, they are precomputed and stored, rather than generated every time they are needed. The temperature data vector $d$ is computed at 21 equally spaced points on the top surface, $x_{1}=a_{i}$ where $a_{i}=-5+\frac{i}{2}$ for $i=0$ to 20 . We then invert the $21 \times 21$ system $M \lambda=d$ to find $\lambda$ and return an estimate of $S$ via equation (4.7). The estimate of $S$ is computed at a suitable number of points on the range of interest, in this case from -5 to 5 . The reconstruction is shown in Figure 2. The dotted line is the actual function $S(x)$ and the solid line is the reconstructed version.

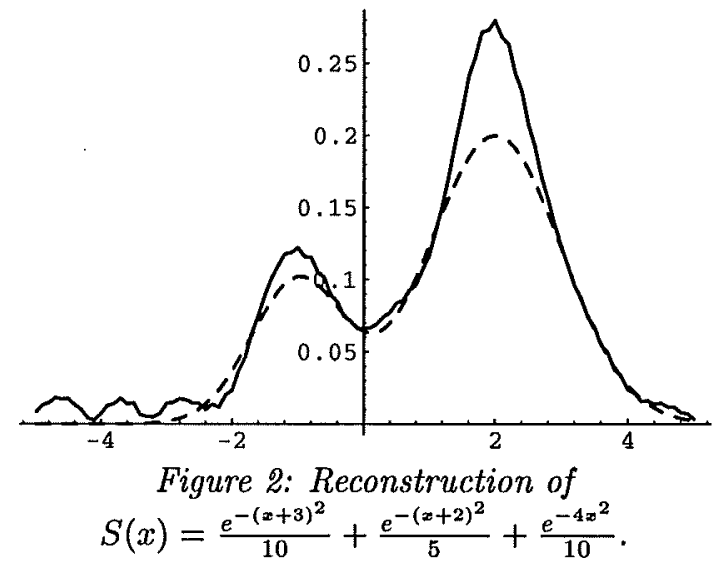

\section{Stability}

Of particular interest is the sensitivity of the inversion procedure with respect to various experimental parameters, e.g., measurement locations. The first task is to quantify the stability or conditioning of the finite data inverse problem. One sensible way to do this is to perform a singular value decomposition on the matrix $M$ defined by equation (4.8) and examine the magnitude of the singular values. When the singular values are small the inversion of $M \lambda=d$ magnifies small perturbations in $d$. Put another way, small singular values mean that relatively large changes in $S$ (and so in $\lambda$ ) produce relatively small changes in the data, so that perturbations in the back surface are "hard to see." Our goal in choosing experimental parameters is therefore to make the singular values of $M$ as large as possible, within certain limits. 
Let us examine how the stability of the inversion procedure depends on the locations of the temperature measurements on the top surface. In the following examples we fix the heating frequency at $\omega=1$ and take measurements of the resulting temperature at 21 equally spaced locations on the interval $[-a, a]$ for several values of $a$. The resulting measurement locations are therefore of the form $a_{i}=-a+\frac{i}{10} a$ for $i=0, \ldots, 20$. In each case the matrix $M$ is computed and a singular value decomposition is performed. Let the singular values of $M$ be denoted by $\alpha_{i}, i=1$ to 21 , arranged in descending order. In Figure 3 we plot the quantity $\log _{10}\left|\alpha_{i}\right|$ versus $i$ for the cases $a=1,2,3,5,10$.

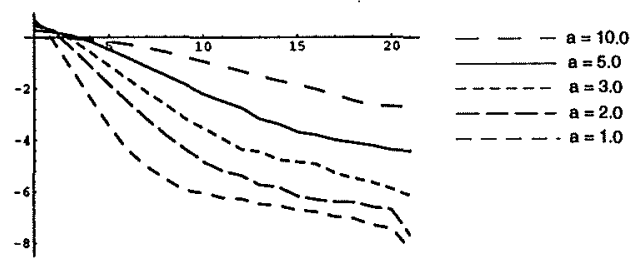

Figure 3: $\log _{10}\left|\alpha_{i}\right|$ versus $i$ for various values of $a$.

It is apparent that as the measurement locations become more spread out (as $a$ gets larger) the singular values decay more slowly and hence the inversion procedure becomes more stable. In light of stability results this is not surprising. When the measurement locations are close together we are able to resolve higher spatial frequencies in the data and so we are able to estimate higher frequencies in the Fourier decomposition of $S$. But according to the stability results these are exactly the portions of $S$ that are difficult to reconstruct-they are heavily damped out in the data. The finite data version of the problem reflects this, with a full 6 orders of magnitude variation for the smallest singular values between the cases $a=1$ and $a=10$.

Another way to look at the stability of the various experimental configurations is to suppose that we have an "error magnification tolerance" $E$, and that in the inversion procedure we disregard all singular vectors whose singular values are less than $\frac{1}{E}$. The inversion procedure is then stabilized at the expense of rendering those components of $S$ lying in the span of the corresponding functions invisible. Figure 4 shows the number of singular values of $M$ which satisfy $\alpha_{k}>\frac{1}{E}$ versus $\log _{10}(E)$ for $E$ from 1 to $10^{-9}$. As in the previous examples, the matrix $M$ is $21 \times 21$ and we use measurement locations on the top surface $a_{i}=-a+\frac{i}{10} a, i=0, \ldots, 20$ for $a=1,2,3,5,10$. The heating frequency is $\omega=1$.

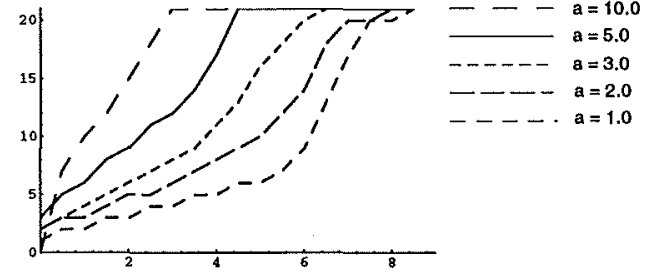

Figure 4: Number of singular values with $\alpha_{i}>\frac{1}{E}$ versus $\log _{10}(E)$ for various values of $a$.

Figure 4 also makes clear that as the measurement locations become spread out more singular values satisfy $\alpha_{i}>\frac{1}{E}$. The inversion procedure then admits more basis functions, presumably improving the fidelity of the reconstruction. In the two cases below we perform the actual reconstruction with $E=100$ (so only singular values greater than 0.01 are admissible) and add a small amount of random noise to the data (equal to 10 percent of the maximum signal strength). We then perform a reconstruction which omits all basis vectors whose corresponding singular values are less than $\frac{1}{E}$. Figure 5 illustrates the case in which the measurements locations are equally spaced from -5 to 5 ; there are 9 admissible singular values.

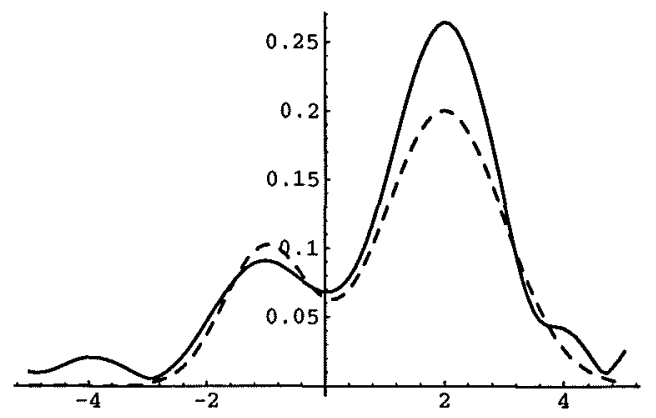

Figure 5: Reconstruction of $S(x)$ for 21 measurements on $[-5,5]$, tolerance $E=10^{2}$.

In Figure 6 we take the 21 measurements on the smaller interval $[-1,1]$, which yields only 3 admissible singular values.

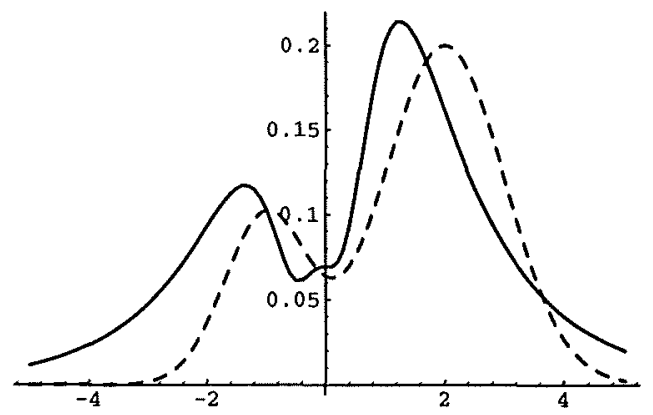

Figure 6: Reconstruction of $S(x)$ for 21 measurements on $[-1,1]$, tolerance $E=10^{2}$. 
The reconstruction in Figure 6 is noticeably inferior to that of Figure 5, but we have only 3 admissible basis functions with which to construct $S(x)$. Increasing the value of $E$ to admit more basis functions is not successful. Figure 7 illustrates what happens if we take $E=10^{4}$ with measurements on $[-1,1]$. Now 5 singular values are admissible, but the reconstruction is overwhelmed by noise.

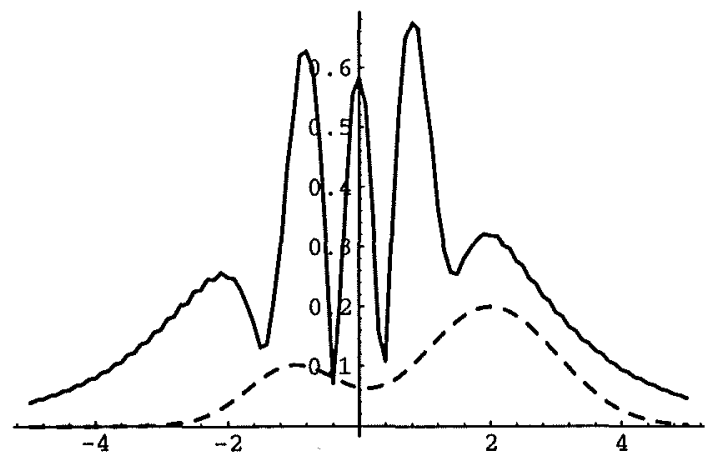

Figure 7: Reconstruction of $S(x)$ for 21 measurements on $[-1,1]$, tolerance $E=10^{4}$.

The moral seems clear: for maximum stability with a fixed number of measurement locations, we should spread the measurements over as large a region as possible. There are limits to this approach, however. If we spread out the measurements we do gain stability, but we will no longer be able to estimate high frequencies in the Fourier decomposition of $S$. This is illustrated by Figure 8 , where we take 21 noise-free measurements on the interval $[-10,10]$ and estimate $S$ with error tolerance $E=10^{2}$. In this case all of the singular values are admissible.

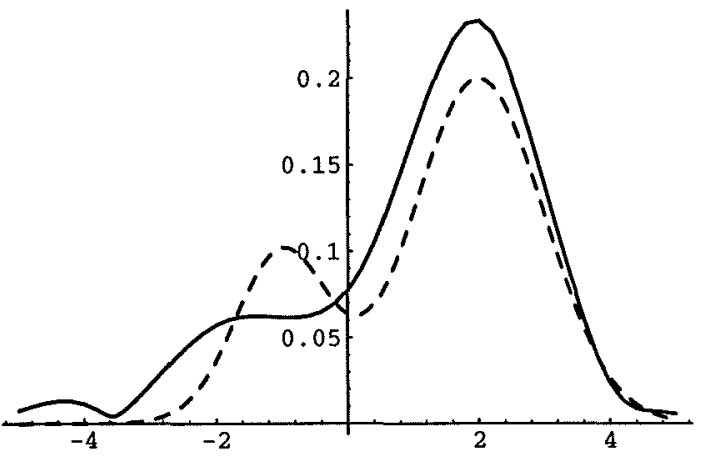

Figure 8: Reconstruction of $S(x)$ for 21 measurements on $[-10,10]$, tolerance $E=10^{2}$.

Despite the fact that the inversion is quite stable, our inability to resolve high frequencies results in a loss of resolution of small-scale detail in the reconstruction. With regard to the distribution of the measurement locations, the reconstruction process involves a compromise between stability and resolution of small-scale features. If the data points are too closely spaced, the inversion procedure is unstable. If the data points are too spread out, the inversion procedure becomes stable, but resolution is lost; measurements taken far from the support of the defect contain little information, because the heat diffuses very rapidly. How shall we find the "best" spacing for measurements? One useful possibility is to incorporate a priori information or assumptions into the inversion procedure. We will illustrate the idea by examining the problem under the assumption that the defect or function $S$ is supported in a known interval.

In the following examples we assume that the defect being imaged is supported in the interval $[-2,2]$. The only modification to the inversion procedure is that the matrix $M$ is computed in accordance with equation (4.9) and the function $S$ is estimated using equation (4.10). We will study the stability of the inversion procedure with respect to the distribution of the measurement locations on the top surface.

As in the previous cases, we choose measurement locations at $x_{1}=a_{i}$ on the sample top surface, where $a_{i}=-a+\frac{i}{10} a$ for $i=0$ to 20 . The heating frequency in all cases that follow is $\omega=1$. Let us begin by examining the singular values of the inversion matrix $M$ for a few choices of $a$. In Figure 9 we plot the quantity $\log _{10}\left|\alpha_{i}\right|$ versus $i$ for $a=0.5,1.0,2.0,5.0,10.0$.

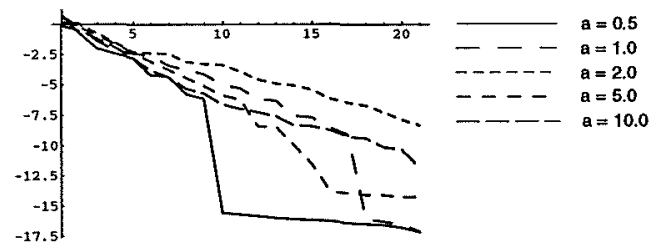

Figure 9: Singular values $\alpha_{i}$ versus $i$ for various values of $a$.

The figure shows that the best conditioning for the inverse problem occurs at $a=2$, when the measurement locations are distributed approximately in the same interval in which the defect is assumed to be supported. As before, closely spaced locations give rise to an illconditioned problem. However unlike the previous cases widely spaced nodes also result in poor conditioning. When $M$ is computed using equation (4.9) those rows of $M$ corresponding to measurement locations far from the support of $S$ are very nearly set to zero since the function $c\left(x-a_{i}\right)$ is rapidly decreasing away from $a_{i}$.

If an error magnification tolerance $E$ is specified, we can plot the number of allowable singular values $\alpha_{i}>\frac{1}{E}$ versus $\log _{10}(E)$ for the different node spacings. 


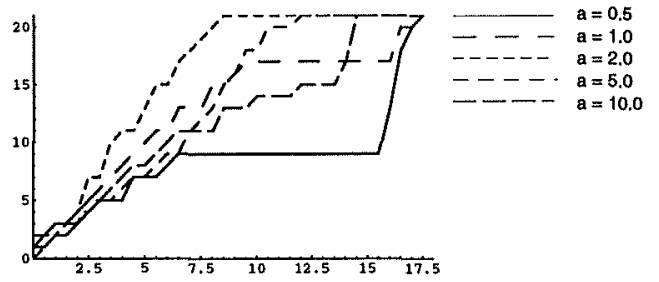

Figure 10: Number of singular values with $\alpha_{i}>\frac{1}{E}$ versus $\log _{10}(E)$ for various values of $a$.

As expected, $a=2.0$ allows more singular values for a fixed value of $E$ than any other choice for measurement spacing. It is useful to look at a few reconstructions based on this strategy. In the two cases below we take $E=300$ (so only singular values greater than $\frac{1}{300}$ are admissible) and add a small amount of random noise to the data (equal to 10 percent of the maximum signal strength). We then perform a reconstruction which omits all singular values less than $\frac{1}{E}$. The function defining the back surface is $S(x)=$ $\frac{1}{10} e^{-2(x+1)^{2}}+\frac{1}{5} e^{-3(x-1)^{2}}$. Figure 11 illustrates the first case using $a=2$, the best choice according to Figure 10. In this case 7 singular values are admissible.

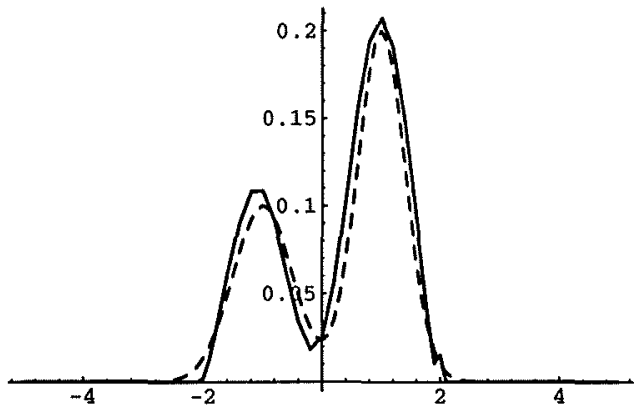

Figure 11: Reconstruction of $S(x)$ for 21 measurements on $[-2,2]$, tolerance $E=300$.

For $a=10$ we have 4 admissible singular values and the reconstruction shown in Figure 12.

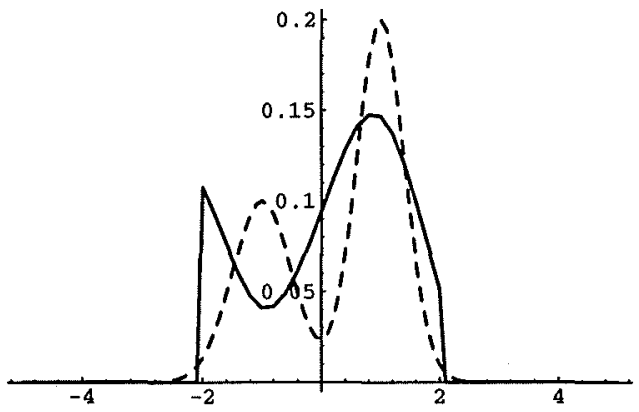

Figure 12: Reconstruction of $S(x)$ for 21 measurements on $[-10,10]$, tolerance $E=300$.

The case $a=0.5$ also yields 4 admissible singular values and the reconstruction shown in Figure 13.

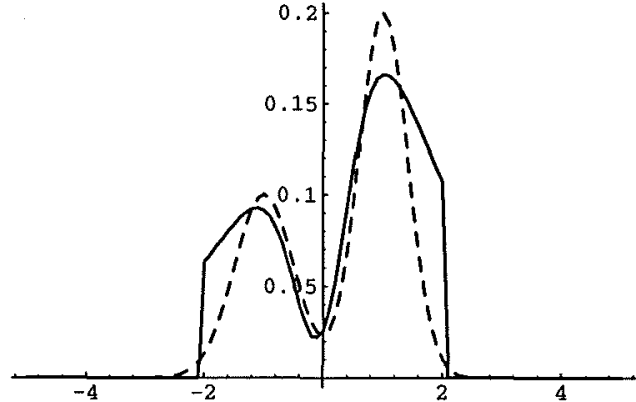

Figure 13: Reconstruction of $S(x)$ for 21

measurements on $[-0.5,0.5]$, tolerance $E=300$.

The actual reconstructions confirm that $a=2$ yields the most desirable results. Choosing $a$ significantly smaller or larger than the support of $S$ results in decreased stability and/or accuracy for the reconstruction.

Of course, the assumption that $S$ is supported in a given interval should be detrimental to the reconstruction if that assumption turns out to be false. In the following case we let $S(x)=\frac{1}{10} e^{-2(x+1)^{2}}+\frac{1}{5} e^{-3(x-4)^{2}}$ and perform the reconstruction under the assumption that $S$ is supported in the interval $[-2,2]$. We take measurements at 21 equally spaced location between -2 and 2, the best case from above, and use an error tolerance $E=300$. The result is shown in Figure 14 .

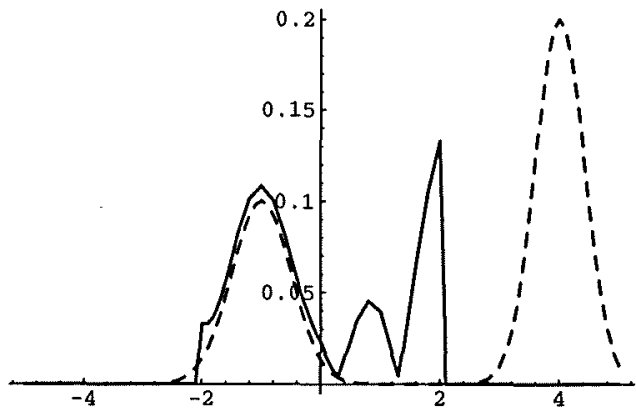

Figure 14: Reconstruction of $S(x)$ for 21 measurements on $[-2,2]$, tolerance $E=300$.

The incorrect assumption obviously introduces errors into the reconstruction, although that portion of $S$ which is non-zero in the interval $[-2,2]$ is still recovered with reasonable accuracy.

\section{Concluding Remarks}

In this paper we have investigated the inverse problem of recovering an unknown boundary portion of some object by applying a heat flux to an accessible portion of the boundary and measuring the resulting temperature response. We have considered a linearized version of the problem and found that the continuous version of the inverse problem, in which one has data at every point 
on the accessible portion of the surface, is extremely ill-posed. Indeed, the linearized version requires one to solve a first kind convolution integral equation for the unknown surface. The convolution kernel has a Fourier transform which dies rapidly at infinity, and so the inversion is extremely sensitive to the data at high spatial frequencies. We performed a variety of numerical studies which show that the ill-posedness is directly reflected in the finite data version of the problem, by the rapid decay of the singular values of the matrix which governs the inversion process. This ill-posedness depends on a number of factors; in particular, the locations of the measurements have a large effect on the conditioning of the inverse problem, and these effects mirror the behavior of the continuous version. We have also considered the effect of including a priori assumptions in the finite data inversion procedure, by weighting appropriate Hilbert spaces in which the solution $S$ resides. The inclusion of this information can help in determining the optimal locations for measurements on the top surface.

There are a number of interesting directions we could take from here. In our studies we used only the input flux whose magnitude is identically one on the top surface. Similar results can be obtained for more general fluxes, and this would allow one to study the effect that the input heat flux has on sensitivity and resolution. The fully time-dependent case would also be of interest. The procedure presented in this paper would also work for a full three- dimensional problem, although qualitatively the results should be the same-the high spatial frequencies in the back surface should be difficult to see.

As mentioned earlier, the inversion process which chooses that function with minimal $L^{2}$ norm which is consistent with the measured data seems to act like a form of regularization for the inverse problem. It would be interesting to examine in what sense this is true, and how it relates to more traditional forms of regularization. It is also possible (and not difficult) to carry out the same minimization process in higher Sobolev spaces, e.g., $H^{1}$, and thus put a higher "penalty" on functions with oscillations. This too would make an interesting study. We would also like to examine conditions under which our inversion procedure is guaranteed to converge to the solution of the linearized inverse problem.

\section{References}

[1] Abramowitz, Milton and Irene Stegun, Handbook of mathematical functions, Applied Mathematics Series, vol. 55. Washington: National Bureau of Stan- dards, 1964. Reprinted 1968 by Dover Publications, New York.

[2] Atkinson, K.E., A survey of numerical methods for the solution of fredholm integral equations of the second kind, SIAM, Philadelphia, PA, 1976.

[3] Banks, H.T. and F. Kojima, Boundary shape identification problems in two-dimensional domains related to thermal testing of materials, Quart. Appl. Math., Vol. 47 (1989), pp. 273-293.

[4] Banks, H.T., F. Kojima and W.P. Winfree, Boundary estimation problems arising in thermal tomography, Inverse Problems 6 (1990), pp. 897-922.

[5] Bryan, K. and L. Caudill Jr., An Inverse Problem in Thermal Imaging, ICASE report 94-99, submitted to SIAM J. Appl. Math.

[6] Byrne, Charles L. and Raymond M. FitzGerald, Reconstruction from partial information with applications to tomography, SIAM J. Appl. Math. Vol. 42, no. 4, (1982), pp. 933-940.

[7] Gisser, D.G., D. Isaacson, and J.C. Newell, Electric current computed tomography and eigenvalues, SIAM J. Appl. Math, Vol 50, no. 6, (1990), pp. 1623-1634.

[8] D. Isaacson, and M. Cheney, Effects of measurement precision and finite numbers of electrodes on linear impedance imaging algorithms. 1990, preprint.

[9] Dobson D. and F. Santosa, Stability and resolution analysis of an inverse problem in electrical impedance tomography-dependence on the input current patterns. SIAM J. Appl. Math. Vol 54, no. 6, (1994), pp. 1542-1560.

[10] Patel, P.M., S. K. Lau and D.P. Almond, A review of image analysis techniques applied in transient thermographic nondestructive testing, Nondestructive Testing and Evaluation, Vol. 6 (1992), pp.343364.

[11] Tricomi, F.G. Integral equations. New York: Interscience Publishers, 1957.

[12] Wing, G. Milton A primer on integral equations of the first kind: the problem of deconvolution and unfolding. SIAM, 1991. 